\title{
Herbicides Registered for Pine Management in Florida - 2005 Update $^{1}$
}

\author{
Anna Osiecka, Jarek Nowak, Alan Long, and Mark Mossler²
}

Forests cover 16.2 million acres or $47 \%$ of Florida's total land area and have a total economic impact of $\$ 16.5$ billion annually (Hodges et al., 2005). About ninety percent of Florida forestland, or 14.7 million acres is commercially productive timberland. Pine forests account for 50\%, and pine plantations for $32 \%$ of Florida's timberland, which is the highest proportion of planted pine among all 13 southern states. Florida is an important contributor to the U.S. timber economy, with annual roundwood production of 607 million cubic feet, or $3 \%$ of the U.S. total (Howard, 2001).

Most of the pine plantations in Florida and throughout the South are intensively managed to meet the growing demand for wood fiber, pine straw and other forest products. In addition, increasing interest in native ecosystem restoration and wildlife habitat are generating new management challenges. One of the major components of successful pine forest management is vegetation management. Herbicide use for vegetation management in forestry has increased dramatically during the last 25 years. Efficient, safe and environmentally sound herbicide use requires an up-to-date knowledge of weed-control technology. New herbicides are constantly being developed and registered for use in Florida. At the same time, production of older herbicides might be discontinued, or their registration in the state of Florida terminated.

Herbicides are used for a variety of applications in managing pine forests to control or alter undesirable vegetation. Site preparation is one use where the current vegetation can be controlled by herbicides prior to planting pine seedlings. After planting, herbaceous weed control with herbicides can aid the survival and growth of the young pine seedlings during establishment phase. In older pine stands herbicides can be applied to release established pine trees from competing woody plants. Individual stems of undesirable vegetation can be treated with herbicides throughout the pine rotation, from site preparation to harvest. Herbicides could

1. This document is CIR1475, one of a series of the School of Forest Resources and Conservation, Florida Cooperative Extension Service, Institute of Food and Agricultural Sciences, University of Florida. First published in April 2005. Please visit the EDIS website at http://edis.ifas.ufl.edu.

2. Anna Osiecka, Biological Scientist and Jarek Nowak, Assistant Professor, Extension Specialist, Forestry, North Florida Research and Education Center-Quincy; Alan Long, Associate Professor, School of Forest Resources and Conservation; and Mark Mossler, Pesticide Information Specialist, Pesticide Information Office, Agronomy Department, Cooperative Extension Service, Institute of Food and Agricultural Sciences, University of Florida, Gainesville, FL 32611.

The use of trade names in this publication is solely for the purpose of providing information. UF/IFAS does not guarantee or warranty the products named, and references to them in this publication do not signify our approval to the exclusion of other suitable products. The products included in tables 1 and 2 were listed as "active registrations" in FDACS Online Pesticide System as of March 24, 2005. FDACS is the sole authority on the herbicides registered in Florida.

The Institute of Food and Agricultural Sciences (IFAS) is an Equal Opportunity Institution authorized to provide research, educational information and other services only to individuals and institutions that function with non-discrimination with respect to race, creed, color, religion, age, disability, sex, sexual orientation, marital status, national origin, political opinions or affiliations. U.S. Department of Agriculture, Cooperative Extension Service, University of Florida, IFAS, Florida A. \& M. University Cooperative Extension Program, and Boards of County Commissioners Cooperating. Larry Arrington, Dean 
also be used to remove certain vegetation types to favor another, especially in the case of restoring native habitats. Some herbicides are registered for applications in wetlands or aquatic areas in the forests.

This publication presents a list of herbicides registered for forestry use in Florida (Table 1) and provides information on their basic weed control characteristics and silvicultural applications (Table 2). All herbicide active ingredients important in pine management in Florida are included. Others, that are registered in Florida, but are rather marginally used for pine plantations, like 2,4-D, atrazine, dicamba, paraquat, and simazine, are also listed. Since an increasing number of private and public owners manage their forestlands for non-commodity purposes, herbicides that are intended mostly for turf and/or landscape maintenance, but also labeled for forestry, have been included as well.

Most of the active ingredients are available in different formulations from different manufacturers and/or distributors under a variety of trade names. It is beyond the scope of this publication to mention all of the silvicultural herbicides available on the market. On the other hand, it seems useful to give a few examples of commercial products for each active ingredient. It has to be emphasized that there might be considerable differences even among herbicides with the same concentration of the same active ingredient(s) resulting, for example, from differences in adjuvants or solvents. Therefore, before making any herbicide substitutions, one has to be sure that the selected product is appropriate for the intended application. Inclusion of a product trade name, or a company name in this publication does not constitute an endorsement of a product or a company, as other products manufactured by different companies might be equally suited for the intended herbicide use.

The examples of herbicides registered for pine management in Florida are listed alphabetically, first by active ingredient and then by trade name (Table 1). Products with the same Environmental Protection Agency (EPA) registration number are grouped together. Herbicides containing more than one active ingredient are placed according to the highest percentage of active ingredient. Herbicide terms used in Table 1 and on herbicide labels are defined as follows:
Active ingredient (a.i.) is the chemical substance that impacts plant metabolism and is designed to negatively affect the undesirable vegetation. The active ingredient has the greatest influence on the properties and behavior of the herbicide and is the primary factor in herbicide selection for a particular application. The exact chemical name of the active ingredient is useful to chemists to ensure precise communication. The common name is a simplified, less technical name of an active ingredient, most often used in herbicide recommendations, technical literature and in Table 1 and Table 2.

Formulation is the commercial preparation of the herbicide, including one or more active ingredients, usually a solvent and, possibly, adjuvant(s), such as surfactants. Each formulation is registered and marketed under a separate trade name. Frequently the same formulation may be marketed under several trade names by different companies or even the same manufacturer or distributor. Such is the case with Dow's Accord ${ }^{\circledR}$ Concentrate, Glypro ${ }^{\mathrm{TM}}$ and Rodeo®, all three of which are the same formulation of glyphosate (53.8\%), registered under the same EPA registration number, but marketed for different applications.

Trade names of herbicides may include numbers and/or letters to further identify the product. The number immediately after the name refers to the concentration of active ingredient. The amount of active ingredient in an herbicide is often expressed as a percentage of volume or mass of the commercial product. For example, Pendulum ${ }^{\circledR} 2 \mathrm{G}$ contains $2 \%$, by weight, of the active ingredient pendimethalin. Labels may additionally provide an acid equivalent (a.e.), which refers to the theoretical yield of a parent acid from an herbicide active ingredient that has been formulated as a derivative, such as an amine or ester. Acid equivalent content is useful especially when comparing herbicides containing different chemical forms of the same active ingredient. For example, Garlon $^{\mathrm{TM}} 3 \mathrm{~A}$ contains $44.4 \%$ of the triethylamine salt of triclopyr, or $31.8 \%$ ( $3 \mathrm{lb} / \mathrm{gal}$ ) of the acid equivalent, triclopyr. Garlon ${ }^{\mathrm{TM}} 4$ contains $61.6 \%$ of butoxyethyl ester of triclopyr, or $44.3 \%$ (4 lb/gal) of the acid equivalent, triclopyr. In this case, the numbers in the trade names refer to the weight of the 
acid equivalent per volume of the product, with the parent acid, triclopyr, being the herbicidally active portion of the formulation.

Letter(s) included in a trade name often (but not always) indicate formulation of the product. Some of the more common acronyms are:

AC - applicator's concentrate

DF - dry flowable

DG - dry granular

E or EC - emulsifiable concentrate

F or FL - flowable or flowable liquid

$\mathrm{G}$ - granular

L - liquid

LV - low volume concentrate

P or PS - pellet

S - soluble concentrate or ready to use solution

SP - soluble powder

ULV - ultra low volume concentrate

W or WP - wettable powder

WDG - water dispersible granule

WSP - water soluble powder

XP - extruded pellet

Documentation associated with each herbicide includes one or more product Label(s) and a Material Safety Data Sheet (MSDS). These documents, prepared by each herbicide manufacturer, contain detailed information about the herbicide product, including trade name, common name(s), chemical name(s), active ingredient(s) concentration(s) and EPA registration number. Product label(s) provide mostly information on the herbicide application, whereas the MSDS characterizes chemical and physical properties of the herbicide and provides information regarding personal and environmental safety. Information and instructions contained in the label must be strictly followed when purchasing, storing, applying or disposing of herbicides. It is a violation of federal law to use any herbicide in a manner inconsistent with its labeling.

Full text labels and MSDSs for most herbicides are accessible through manufacturers web sites and dedicated databases such as those of Crop Data Management Systems (CDMS) available at http://www.cdms.net, or Crop Protection Reference "Greenbook" available at http://www.greenbook.net. Basic searches of these two databases are available to the general public. Subscribers can perform advanced searches of either database by trade name, common name, manufacturer, crop tree species or weed species. The CDMS database can additionally be searched by state. ChemSearch is a restricted use service of the CDMS database, but is available in each county in Florida through Florida Cooperative Extension Service offices on a trial basis until July 2005 with a possibility of continuation beyond this date. One has to keep in mind that while these and other databases are very useful tools, they are usually not complete and vary in accuracy.

Lists of herbicides registered for use in pine management differ from state to state. Only products registered by the Florida Department of Agriculture and Consumer Services (FDACS) can be legally purchased in the state of Florida. The general public can search the FDACS web site at http://www.flpesticide.us or the National Pesticide Information Retrieval System (NPIRS) at http://state.ceris.purdue.edu for active Florida registrations.

Another good source of pesticide related information (including herbicide relevant links) is the Pesticide Information Office (PIO) at the University of Florida, IFAS, accessible through http://pested.ifas.ufl.edu. The PIO has the responsibility of assembling, maintaining and disseminating current pesticide information in the state of Florida. 


\section{References}

CDMS Labels \& MSDS. 2005.

http://www.cdms.net/manuf. (accessed March 2, 2005)

Ferrell, J.A., G.E. MacDonald, B.J. Brecke, and J. Tredaway Ducar. 2005. Trade Name, Common Name and Registrant of Some Herbicides.

SS-AGR-104. Florida Cooperative. Extension Service, IFAS, University of Florida, Gainesville, Fl. $6 \mathrm{pp}$.

Hodges, A.W., W.D. Mulkey, J.R. Alavalapati, D. R. Carter, and C.F. Kiker. 2005. Economic Impacts of the Forest Industry in Florida, 2003. Final Report to the Florida Forestry Association. University of Florida, Institute of Food and Agricultural Sciences, Food and Resource Economics Department and School of Forest Resources and Conservation. $47 \mathrm{pp}$.

Howard, J.L. 2001. U.S. Timber Production, Trade, Consumption, and Price Statistics 1965 1999. United States Department of Agriculture. Forest Service. Forest Products Laboratory. Research Paper FPL-RP-595. 90 pp.

National Pesticide Information Retrieval System (NPIRS). 2005. Purdue University. http://state.ceris.purdue.edu. (accessed February 17, 2005)

Nordby, D.E. and A.G. Hager. Herbicide Formulations and Calculations: Active Ingredient or Acid Equivalent? Weed Fact Sheet. University of Illinois Integrated Pest Management. http://weeds.cropsci.uiuc.edu/extension/extension.htm (accessed March 1, 2005)

Pesticide Products Registered in Florida. Online Pesticide System. 2005. Florida Department of Agriculture and Consumer Services. Division of Agricultural Environmental Services. Bureau of Pesticides. http://www.flpesticide.us. (accessed March 24, 2005) 


\begin{tabular}{|c|c|c|c|c|c|c|c|c|c|c|c|c|c|c|c|}
\hline  & 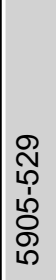 & 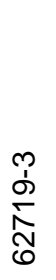 & 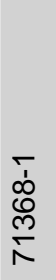 & 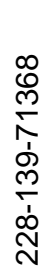 & 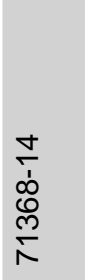 & $\begin{array}{l}\text { ô } \\
+ \\
\dot{0} \\
\text { ○े }\end{array}$ & 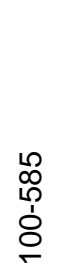 & 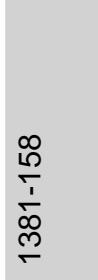 & 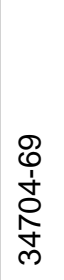 & 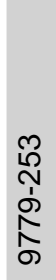 & 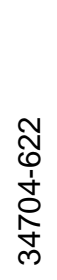 & 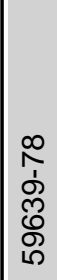 & 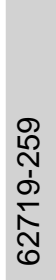 & 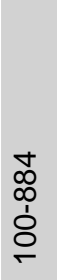 & 옹 \\
\hline 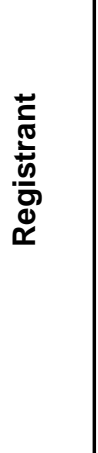 & 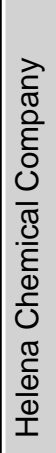 & $\begin{array}{l}0 \\
J \\
0 \\
0 \\
0 \\
\frac{0}{0} \\
.0 \\
0 \\
0 \\
0 \\
\frac{0}{4} \\
3 \\
0\end{array}$ & 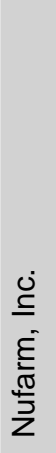 & 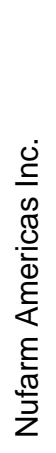 & 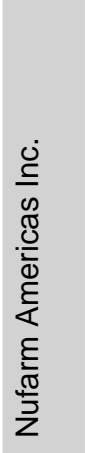 & 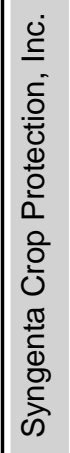 & 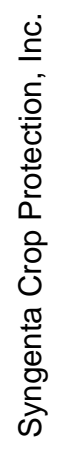 & 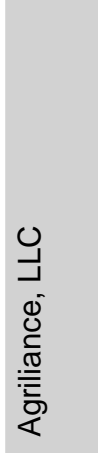 & 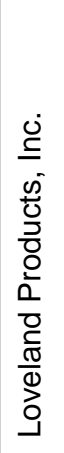 & 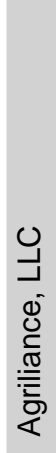 & 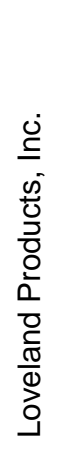 & 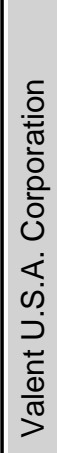 & $\begin{array}{l}0 \\
J \\
0 \\
0 \\
0 \\
0 \\
\frac{0}{0} \\
0 \\
0 \\
0 \\
\frac{0}{0} \\
3 \\
0 \\
0\end{array}$ & 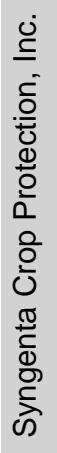 & 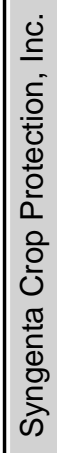 \\
\hline 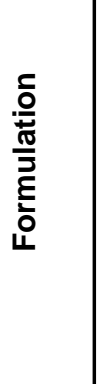 & 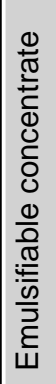 & 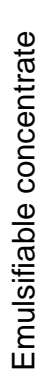 & 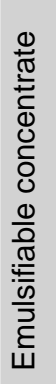 & 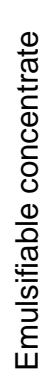 & 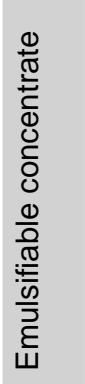 & 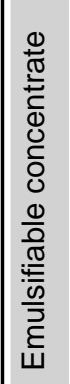 & $\begin{array}{l}\frac{0}{3} \\
\frac{1}{0} \\
\frac{\pi}{0} \\
\frac{0}{0} \\
\frac{0}{0} \\
\frac{0}{0} \\
\frac{0}{0} \\
\frac{0}{0} \\
\frac{0}{0} \\
\frac{\pi}{3} \\
3\end{array}$ & 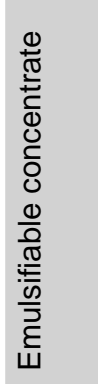 & 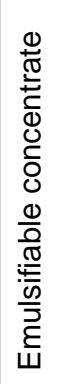 & $\begin{array}{l}\frac{0}{3} \\
\frac{\pi}{\pi} \\
\frac{\pi}{\pi} \\
\frac{0}{0} \\
\frac{0}{0} \\
\frac{0}{0} \\
\frac{0}{00} \\
\frac{0}{0} \\
\frac{1}{0} \\
\frac{\pi}{\pi}\end{array}$ & $\begin{array}{l}\frac{0}{3} \\
\frac{\pi}{\pi} \\
\frac{\pi}{\pi} \\
\frac{0}{0} \\
\frac{0}{0} \\
\overline{0} \\
\frac{0}{00} \\
\frac{0}{0} \\
\frac{0}{0} \\
\frac{\pi}{3}\end{array}$ & 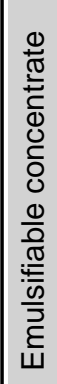 & 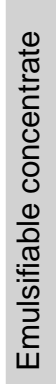 & 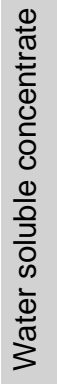 & 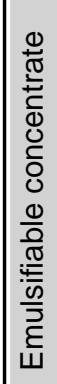 \\
\hline 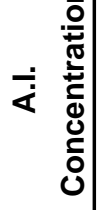 & $\begin{array}{l}\stackrel{0}{\circ} \\
\stackrel{0}{\infty} \\
\stackrel{0}{\circ}\end{array}$ & $\begin{array}{l}\stackrel{\circ}{0} \\
\text { pे } \\
\dot{\varphi} \\
+\end{array}$ & $\begin{array}{l}\stackrel{0}{0} \\
\varnothing \\
\dot{\varphi} \\
\stackrel{f}{*}\end{array}$ & 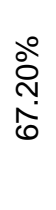 & $\begin{array}{l}\text { ठे } \\
\text { ठे } \\
\text { ஸे }\end{array}$ & 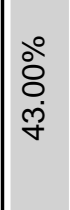 & $\begin{array}{l}\text { ठ̊ } \\
\text { ه̊ं }\end{array}$ & $\begin{array}{l}\stackrel{\circ}{\circ} \\
\text { ஜு }\end{array}$ & 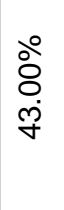 & 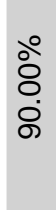 & $\begin{array}{l}\text { ठ̊ } \\
\text { هं }\end{array}$ & $\begin{array}{l}\stackrel{0}{0} \\
\stackrel{0}{0} \\
\stackrel{1}{\sim}\end{array}$ & $\begin{array}{l}\text { ठे } \\
\text { ठे } \\
\text { ᄋं }\end{array}$ & 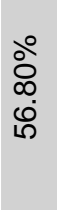 & 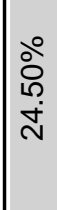 \\
\hline 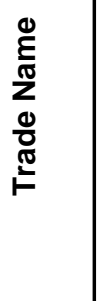 & 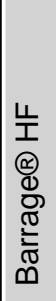 & $\begin{array}{l}\sum_{j} \\
\sum_{0}^{+} \\
\sum_{0}^{+}\end{array}$ & 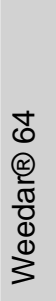 & 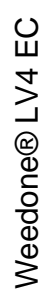 & 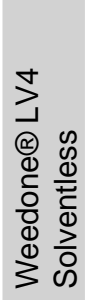 & 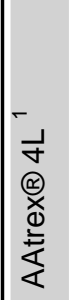 & 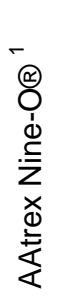 & 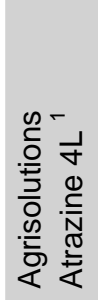 & 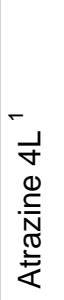 & 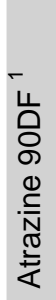 & 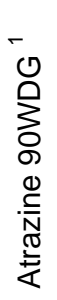 & 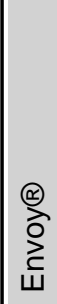 & 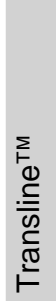 & 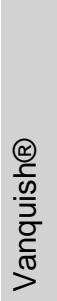 & 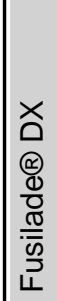 \\
\hline 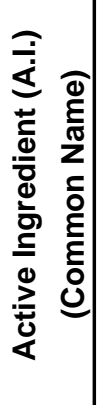 & 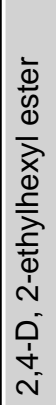 & 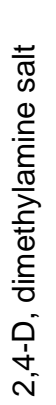 & 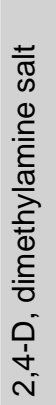 & 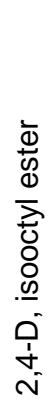 & 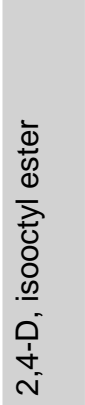 &  & 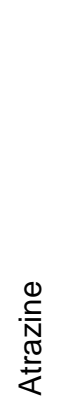 & 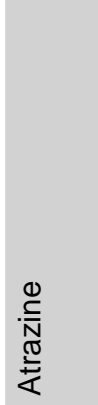 & 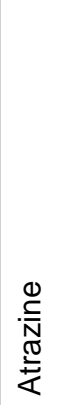 & $\begin{array}{l}\stackrel{\mathscr{N}}{N} \\
\underset{N}{\frac{\pi}{L}}\end{array}$ & $\begin{array}{l}\stackrel{\mathscr{N}}{N} \\
\stackrel{\widetilde{N}}{\frac{\pi}{2}}\end{array}$ & 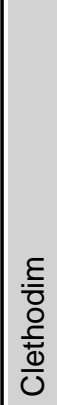 & $\begin{array}{l}\frac{\overline{0}}{\bar{N}} \\
\frac{0}{2} \\
\frac{0}{0} \\
\end{array}$ & 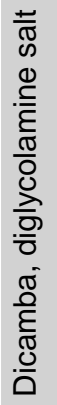 & 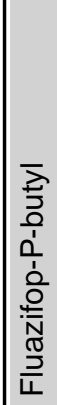 \\
\hline
\end{tabular}







Archival copy: for current recommendations see http://edis.ifas.ufl.edu or your local extension office.

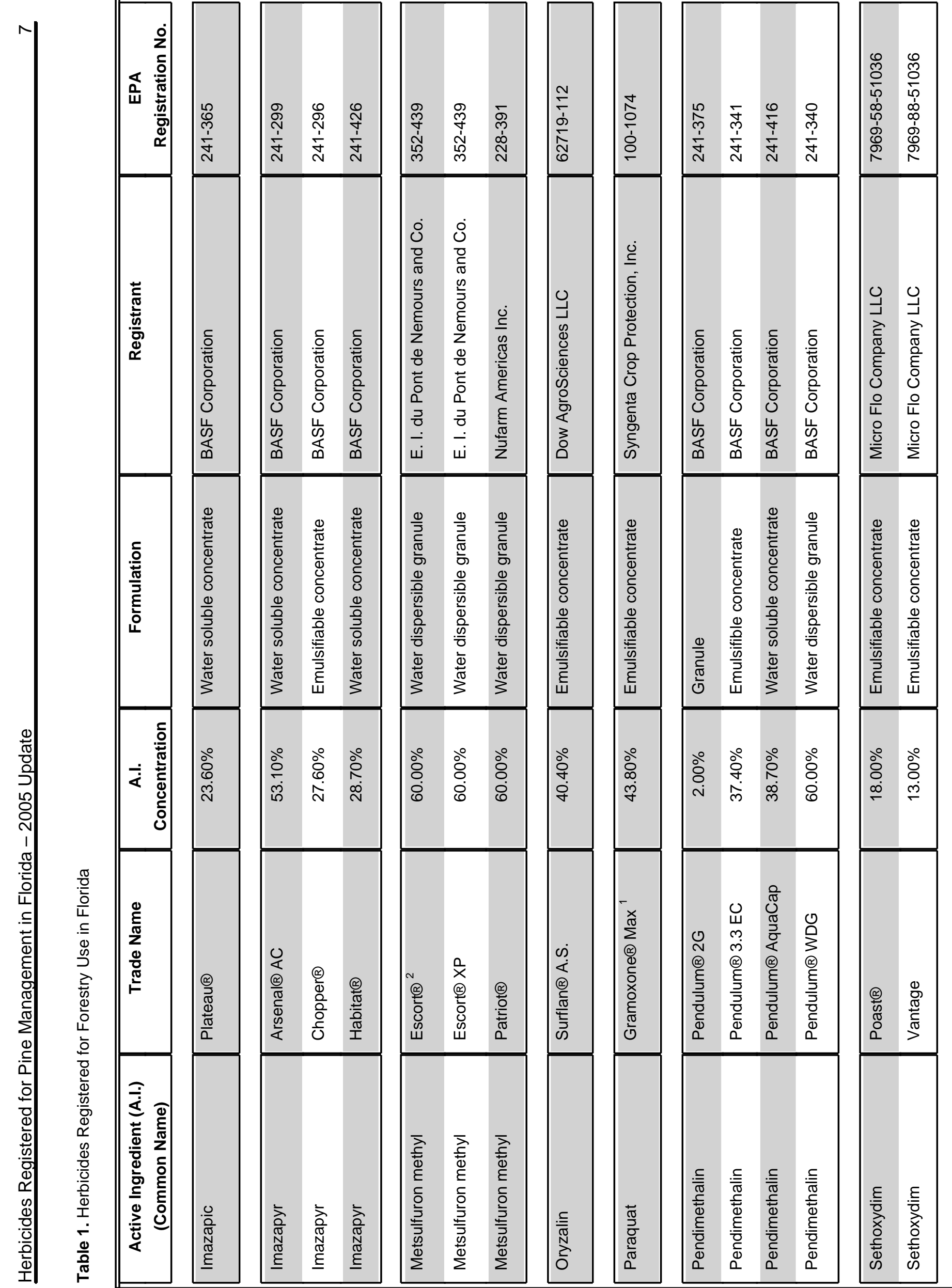




\begin{tabular}{|c|c|c|c|c|c|c|c|c|c|c|c|c|c|}
\hline 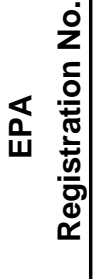 & 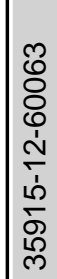 & 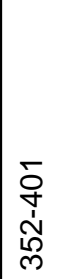 & $\begin{array}{l}\bar{\delta} \\
\grave{1} \\
\text { ஸे } \\
\text { ले }\end{array}$ & $\begin{array}{l}\hat{N} \\
\text { J } \\
\frac{1}{1} \\
\frac{1}{N} \\
\hat{0} \\
\stackrel{N}{N}\end{array}$ &  & $\begin{array}{l}\text { సิ } \\
\vdots \\
\text { ஸે } \\
\text { مै }\end{array}$ & 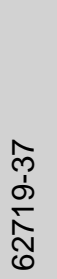 & 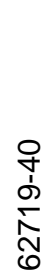 & 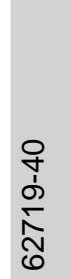 & 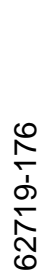 & 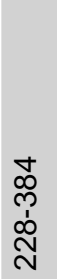 & 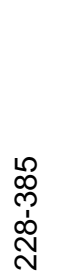 & \\
\hline 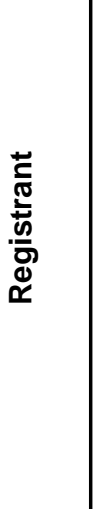 & 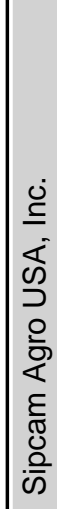 & 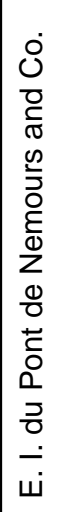 & 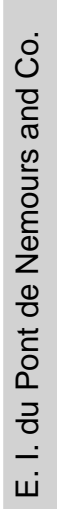 & 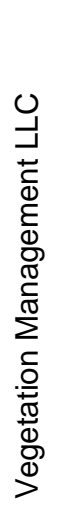 & 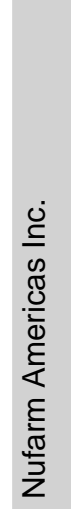 & 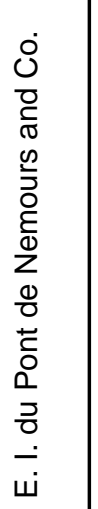 & $\begin{array}{l}0 \\
J \\
0 \\
0 \\
0 \\
\frac{0}{0} \\
\frac{0}{0} \\
0 \\
0 \\
0 \\
\frac{0}{4} \\
0 \\
0\end{array}$ & $\begin{array}{l}0 \\
\\
0 \\
0 \\
0 \\
0 \\
\frac{0}{0} \\
0 \\
0 \\
0 \\
\overline{0} \\
3 \\
0\end{array}$ & $\begin{array}{l}0 \\
\\
0 \\
0 \\
0 \\
0 \\
\frac{c}{0} \\
0 \\
0 \\
0 \\
0 \\
0 \\
3 \\
0 \\
0\end{array}$ & $\begin{array}{l}0 \\
J \\
0 \\
0 \\
0 \\
\frac{0}{0} \\
\frac{0}{0} \\
0 \\
0 \\
0 \\
\overline{0} \\
3 \\
0\end{array}$ & 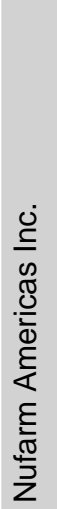 & 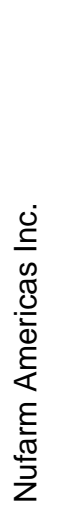 & \\
\hline 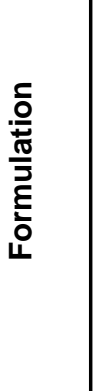 & 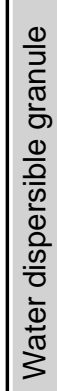 & $\begin{array}{l}\frac{0}{3} \\
\frac{1}{\pi} \\
\frac{\pi}{0} \\
0 \\
\frac{0}{0} \\
\frac{0}{0} \\
\frac{0}{0} \\
\frac{0}{0} \\
\frac{0}{0} \\
\frac{0}{0} \\
\frac{\pi}{\pi} \\
3\end{array}$ & $\begin{array}{l}\frac{0}{5} \\
\frac{\pi}{\pi} \\
\frac{0}{\sigma} \\
\frac{0}{0} \\
\frac{0}{0} \\
\frac{0}{0} \\
\frac{0}{0} \\
\frac{0}{0} \\
\frac{\overline{0}}{\omega} \\
\frac{\pi}{3}\end{array}$ &  & 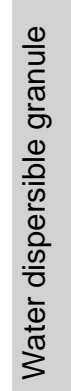 & 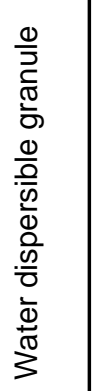 & 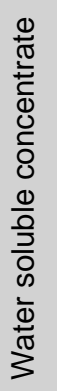 & 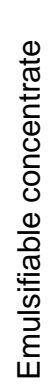 & 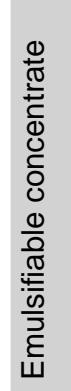 & 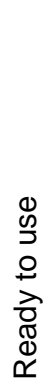 & 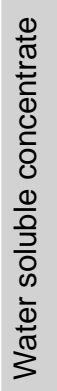 & 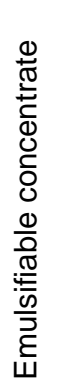 & \\
\hline 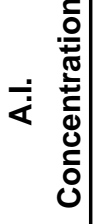 & 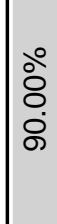 & $\begin{array}{l}\stackrel{0}{\circ} \\
8 \\
\stackrel{\circ}{\circ}\end{array}$ & $\begin{array}{l}\stackrel{\circ}{\circ} \\
\text { ळे }\end{array}$ & $\begin{array}{l}\stackrel{0}{\circ} \\
\text { ठ̊. } \\
\stackrel{\rho}{\circ}\end{array}$ & $\begin{array}{l}\text { ठ̊ } \\
\text { ஜீ } \\
\stackrel{1}{\circ}\end{array}$ &  & $\begin{array}{l}\text { ᄋ̊ } \\
\text { +் } \\
\dot{\forall}\end{array}$ & $\begin{array}{l}\stackrel{0}{0} \\
\dot{0} \\
\dot{0}\end{array}$ & $\begin{array}{l}\stackrel{\circ}{\circ} \\
\dot{0} \\
\dot{\bar{\sigma}}\end{array}$ & 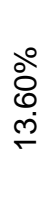 & $\begin{array}{l}\stackrel{0}{O} \\
\text { +े } \\
\dot{\forall}\end{array}$ & $\frac{\stackrel{0}{0}}{\grave{0}}$ & \\
\hline 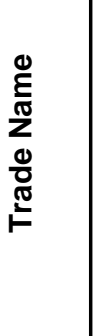 & 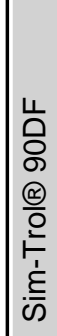 & 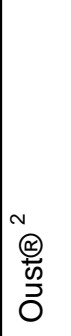 & $\begin{array}{l}\frac{0}{x} \\
\stackrel{9}{9} \\
\frac{5}{3} \\
0\end{array}$ & $\sum_{\substack{\infty \\
\infty}}^{\stackrel{p}{R}}$ & $\frac{\frac{1}{2}}{\frac{0}{0}}$ & 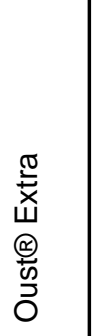 & 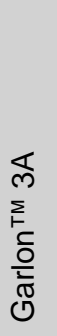 & $\begin{array}{l}\sum_{t}^{+} \\
\sum^{\prime} \\
\frac{0}{\bar{V}} \\
0 \\
0\end{array}$ & 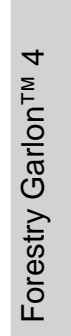 & 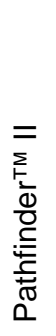 & 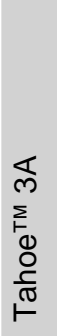 & 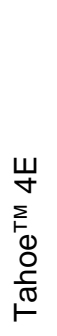 & 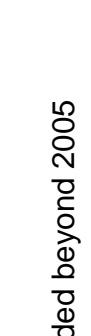 \\
\hline 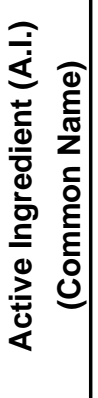 & 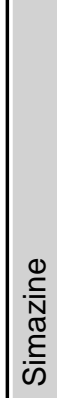 &  & 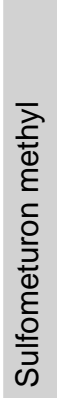 & 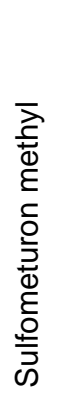 & 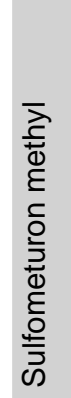 & 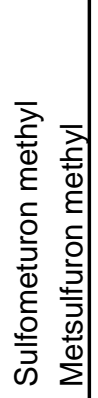 & 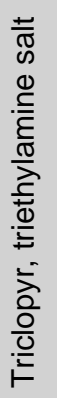 & 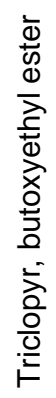 & 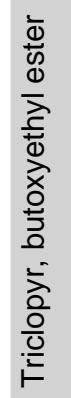 & 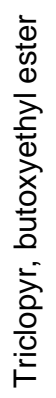 & 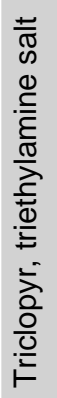 & 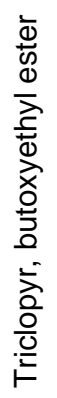 & 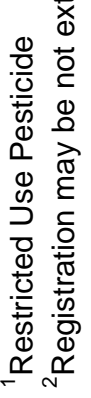 \\
\hline
\end{tabular}




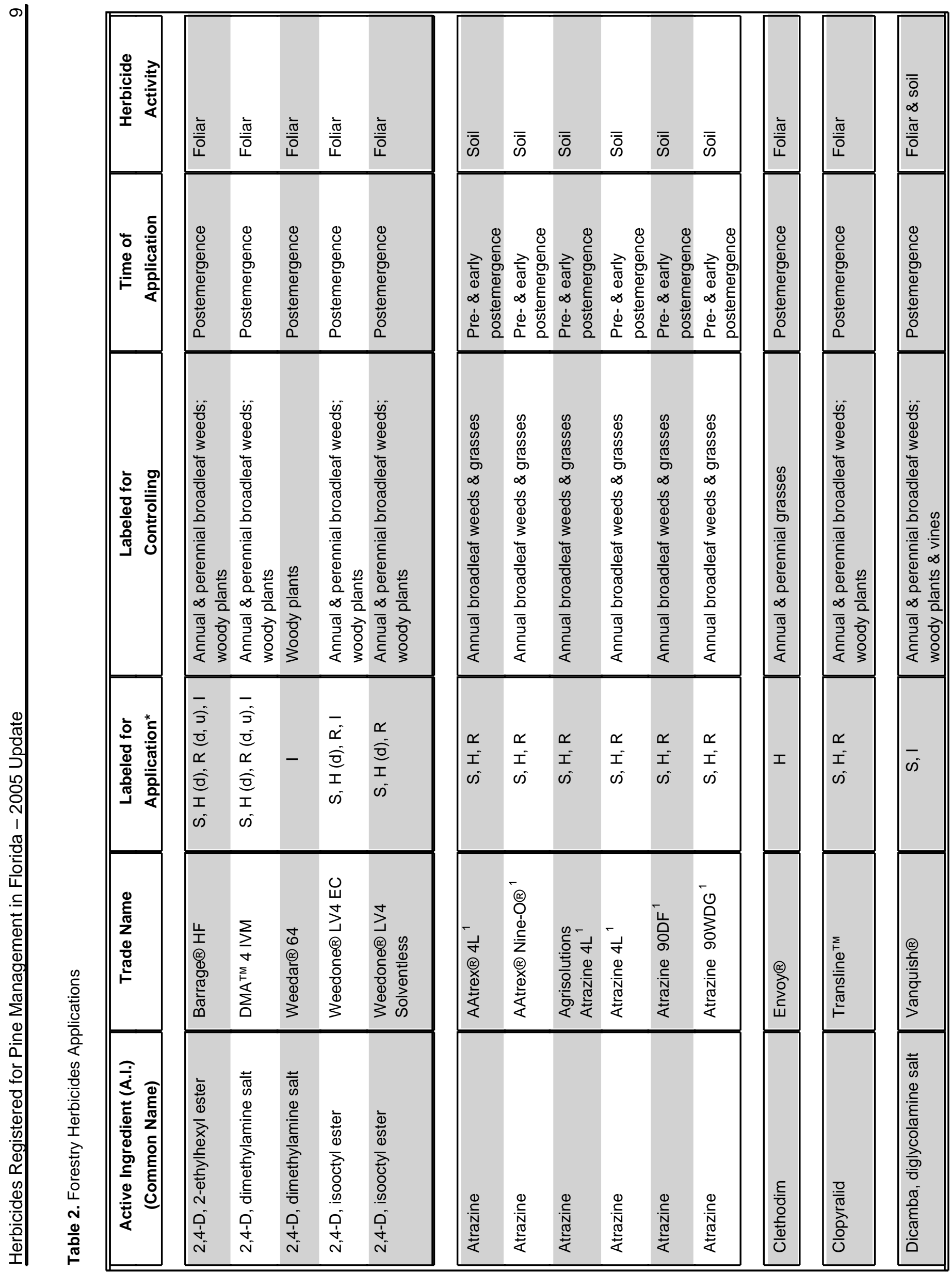




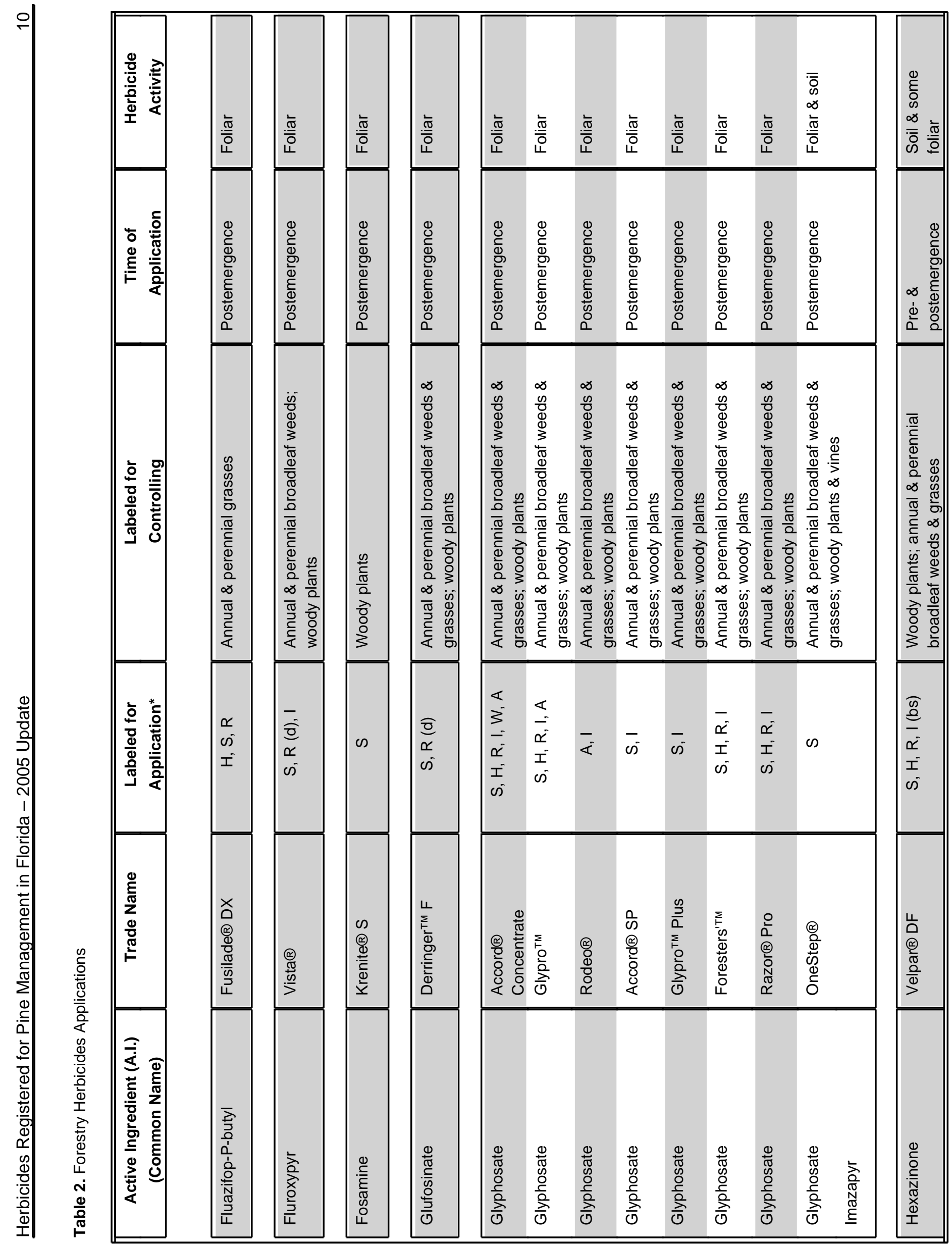




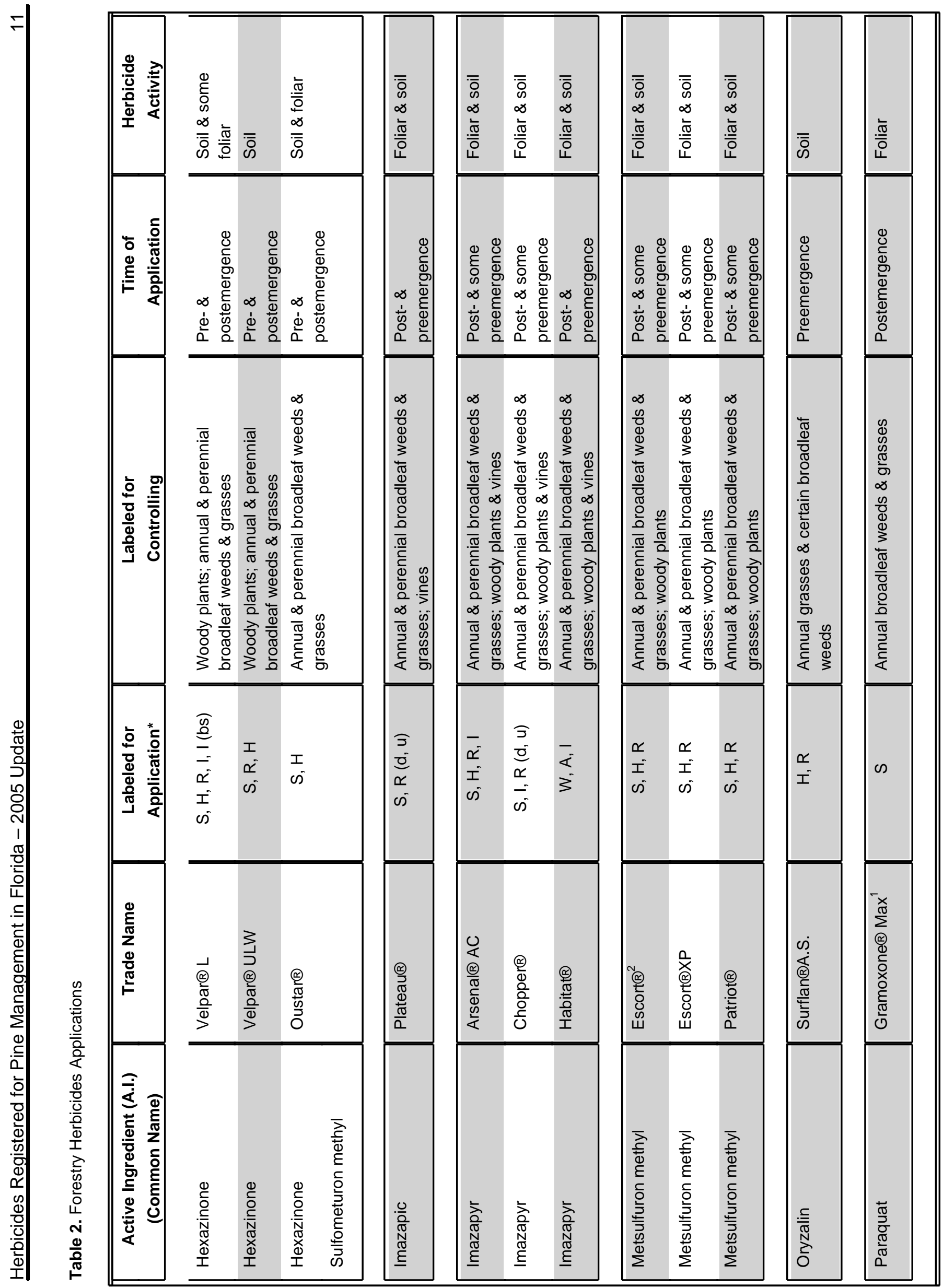




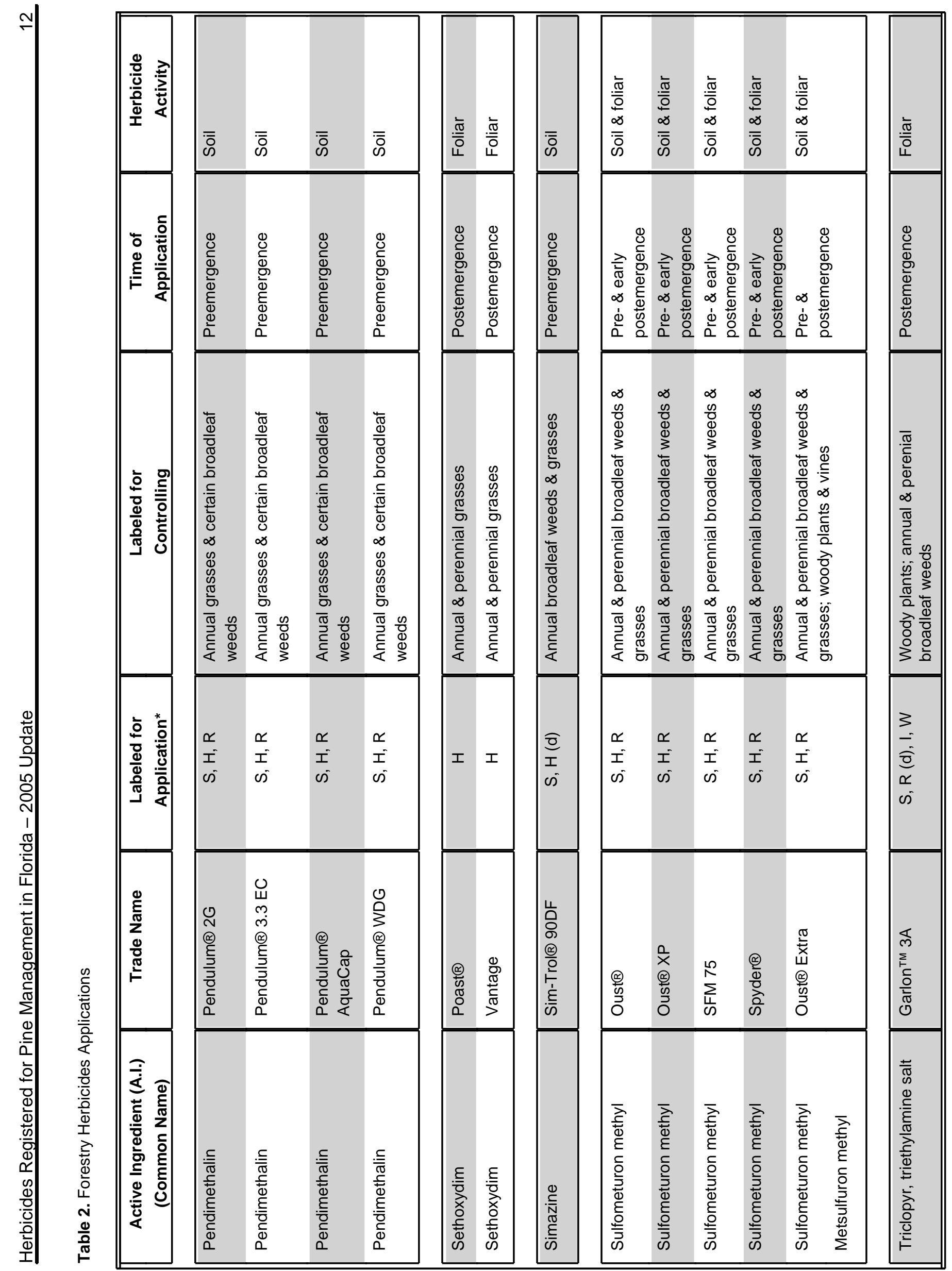




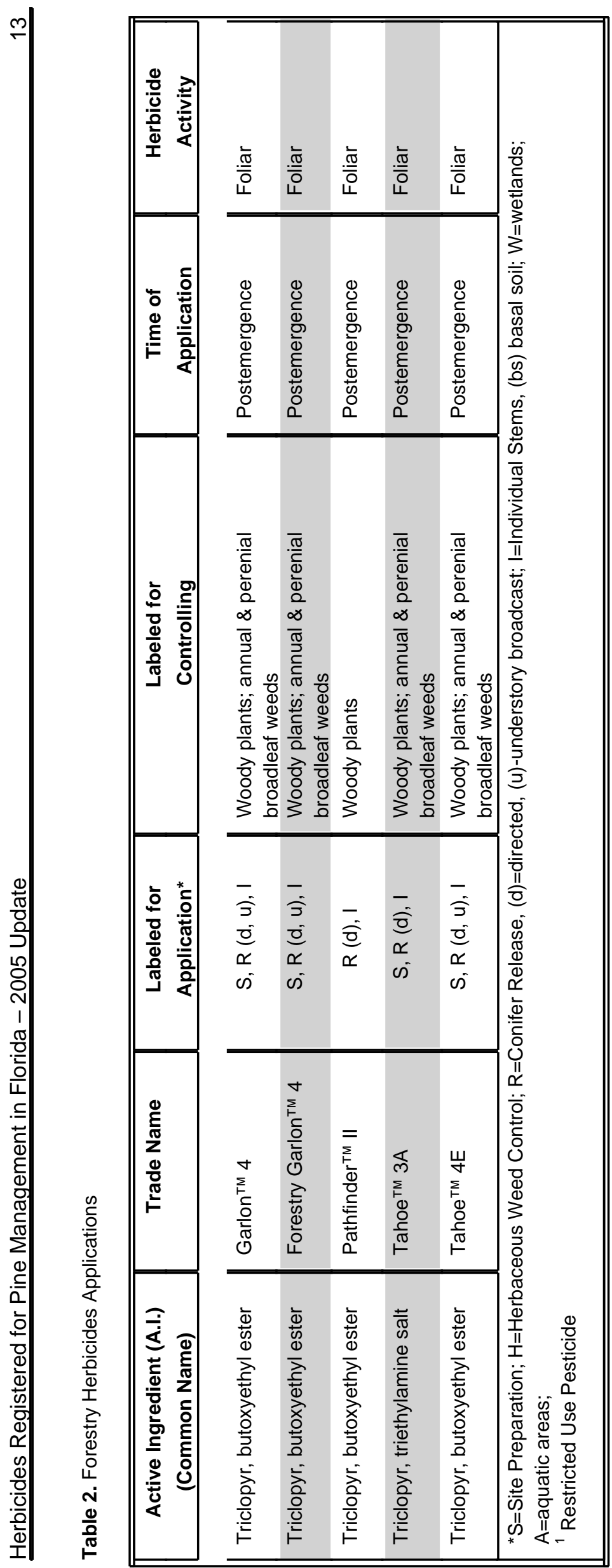

